\title{
The automorphism groups of Zariski open affine subsets of the affine plane
}

\author{
by ZBIGNiEW JELONEK (Kraków)
}

\begin{abstract}
We study some properties of the affine plane. First we describe the set of fixed points of a polynomial automorphism of $\mathbb{C}^{2}$. Next we classify completely so-called identity sets for polynomial automorphisms of $\mathbb{C}^{2}$. Finally, we show that a sufficiently general Zariski open affine subset of the affine plane has a finite group of automorphisms.
\end{abstract}

1. Introduction. The automorphism group of an affine (or more generally non-complete) algebraic variety $X$ is rather difficult to study and only partial results on its structure are known. In [Iit1], [Sak] sufficient conditions for the finiteness of $\operatorname{Aut}(X)$ are given (in terms of logarithmic Kodaira dimension).

Another approach is given in [Jel2] and [Jel3], where we showed that $\operatorname{Aut}(X)$ is finite provided the divisor at infinity of some projective compactification of $X$ is very ample and it does not have uniruled components. Moreover, in [Jel2] we started the study of the automorphism groups of affine Zariski open subvarieties of $\mathbb{C}^{n}$.

This note is a continuation of this study, as well as a continuation of our work on identity sets for polynomial automorphisms (see [Jel1], [Jel3], [Jel5]).

We concentrate on the first non-trivial case, that of the affine plane $\mathbb{C}^{2}$. Our first aim is to give a description of the fixed point set of a non-trivial polynomial automorphism of $\mathbb{C}^{2}$.

We show (Theorem 3.3) that this set is either finite, or a finite union of disjoint plane $\mathbb{C}$-curves. Conversely, for any finite subset $S$ of the plane, or for any finite family $S$ of disjoint plane $\mathbb{C}$-curves we construct an automorphism of the plane for which the set of fixed points is exactly $S$.

1991 Mathematics Subject Classification: 14-01.

Key words and phrases: polynomial automorphisms, the set of fixed points of a polynomial automorphism, the affine plane.

This work was supported by the DFG grant number Ba 423/3-3. 
In particular, we obtain an "if and only if" condition for an affine curve $\Gamma$ to be an identity set for polynomial automorphisms of $\mathbb{C}^{2}$. This generalizes some results of [M-W], [Jel1], [Jel5].

The approach is based on the study of curves $\Gamma \subset \mathbb{C}^{2}$ with infinite group $\operatorname{Stab}_{\Gamma}=\left\{f \in \operatorname{Aut}\left(\mathbb{C}^{2}\right): f(\Gamma)=\Gamma\right\}$. We classify such curves completely (Theorem 3.8).

Finally, we show that for any finite family $\left\{\Gamma_{1}, \ldots, \Gamma_{s}\right\}$ of non-rational curves on the plane, the variety $X:=\mathbb{C}^{2} \backslash \bigcup_{i=1}^{s} \Gamma_{i}$ has a finite automorphism group (Theorem 3.9).

2. Preliminaries. Let us recall some properties of fibres of a primitive polynomial $p$ in two complex variables (see [Suz1], [Suz2], [Z-L], [Zai]).

It is well known that all but finitely many fibres of $p$ are pairwise homeomorphic. Such fibres are called generic. A generic fibre is smooth and irreducible. All other fibres are called degenerate. A point $s \in \mathbb{C}$ for which the fibre $\Gamma_{s}:=p^{-1}(s)$ is generic is called a generic point, otherwise it is degenerate. The set of fibres of a primitive polynomial $p$ will be called a family of curves.

The following proposition was proved in [Suz2] and is crucial to our study:

Proposition 2.1. Let $p: \mathbb{C}^{2} \rightarrow \mathbb{C}$ be a primitive polynomial and let $\chi_{p}$ denote the Euler characteristic of a generic fibre. Let $S$ be the set of all degenerate points. For every $s \in S$ we have $\chi\left(\Gamma_{s}\right)>\chi_{p}$. Moreover,

$$
\sum_{s \in S}\left\{\chi\left(\Gamma_{s}\right)-\chi_{p}\right\}=1-\chi_{p}
$$

We get at once the following interesting

COROllary 2.2. If the generic fibre of a family $p$ is a $\mathbb{C}$-curve (i.e., it is isomorphic to $\mathbb{C}$ ) then all fibres of $p$ are generic (and isomorphic to $\mathbb{C})$. Conversely, if all fibres of a family $p$ are generic then it is a family of $\mathbb{C}$-curves.

If a generic fibre is a $\mathbb{C}^{*}$-curve then the family $p$ has exactly one degenerate fibre $\Gamma_{s}$. Moreover, $\chi\left(\Gamma_{s}\right)=1$.

In the sequel the topological characterization of an irreducible algebraic curve with Euler characteristic 1 will be useful. Let us begin with the following simple observation.

Lemma 2.3. Let $X$ be an algebraic complex curve and let $a \in X$. Then

$$
\chi(X \backslash\{a\})=\chi(X)-1 .
$$

Proof. Consider the triple $\{X \backslash\{a\}, V, V \backslash\{a\}\}$, where $V$ is a small neighbourhood of $a$. More precisely, we can assume that $V$ is a bouquet of $r$ 
discs, where $r$ is the number of components in the germ $X_{a}$. In this situation the point $a$ is a retract of $V$, and $V \backslash\{a\}$ can be retracted to the disjoint sum $\bigcup_{i=1}^{r} S_{i}^{1}$ of $r$ circles. Hence $\chi(V)=1$ and $\chi(V \backslash\{a\})=r \chi\left(S^{1}\right)=0$. Using the Mayer-Vietoris sequence we have

$$
\chi(X \backslash\{a\})+\chi(V)=\chi(X)+\chi(V \backslash\{a\}) .
$$

Thus $\chi(X \backslash\{a\})+1=\chi(X)$.

The simple but useful consequence of the above result is the following

Proposition 2.4. Let $X$ be an irreducible affine curve of genus $g$. (This means that a smooth model $X_{1}$ of a compactification of $X$ has genus $g$. .) Suppose that $X$ has $n$ branches at infinity (i.e., $n$ is the number of points in $X_{1} \backslash X_{0}$, where $X_{0}$ is the normalization of $\left.X\right)$. Let $\operatorname{Sing}(X)=\left\{a_{1}, \ldots, a_{r}\right\}$ be the singular locus. Further, suppose that the germ $X_{a_{i}}$ has $k_{i}$ irreducible components. Then

$$
\chi(X)=2(1-g)-n-\sum_{i=1}^{r}\left(k_{i}-1\right) .
$$

In particular, if $\chi(X)=1$ then $X$ is homeomorphic to $\mathbb{C}$.

Proof. Let $\pi: X_{0} \rightarrow X$ be the normalization. Then $\pi$ is an isomorphism outside $A:=\operatorname{Sing}(X)$, and $\pi^{-1}(A)$ has $\sum_{i=1}^{r} k_{i}$ points. Hence by the lemma

$$
\begin{aligned}
\chi(X) & =\chi(X \backslash A)+r=\chi\left(X_{0} \backslash \pi^{-1}(A)\right)+r=\chi\left(X_{0}\right)-\sum_{i=1}^{r} k_{i}+r \\
& =\chi\left(X_{1}\right)-n-\sum_{i=1}^{r}\left(k_{i}-1\right)=2(1-g)-n-\sum_{i=1}^{r}\left(k_{i}-1\right) .
\end{aligned}
$$

Since $X$ is affine we have $n>0$ and the case $\chi(X)=1$ is possible only if $g=0$ and $n=1$ and all $k_{i}=1$, i.e., if $X$ is homeomorphic to $\mathbb{C}$.

Plane curves homeomorphic to the complex line have a very nice description due to Zằdenberg and Lin (see $[\mathrm{Z}-\mathrm{L}])$ :

Proposition 2.5. Let $X \subset \mathbb{C}^{2}$ be an affine algebraic curve homeomorphic to the complex line. Then in suitable coordinates $X$ can be written as

$$
X=\left\{(x, y) \in \mathbb{C}^{2}: x^{k}=y^{l},(k, l)=1\right\} .
$$

Remark 2.6. The proposition above is a generalization of the famous Abhyankar-Moh-Suzuki theorem (see [A-M], [Suz1]):

If $\Gamma \subset \mathbb{C}^{2}$ is a curve isomorphic to $\mathbb{C}$ then in some coordinates we have $\Gamma=\left\{(x, y) \in \mathbb{C}^{2}: x=0\right\}$.

There is the following useful consequence of the above considerations. 
Proposition 2.7. Let $p: \mathbb{C}^{2} \rightarrow \mathbb{C}$ be a family of curves. If there is only one degenerate fibre $\Gamma$ in $p$ and $\Gamma$ is irreducible then in some coordinates

$$
\Gamma=\left\{(x, y) \in \mathbb{C}^{2}: x^{k}=y^{l},(k, l)=1\right\} .
$$

Pr o of. Indeed, by Proposition 2.1 the curve $\Gamma$ has Euler characteristic equal to 1 . Hence by Proposition 2.4 it is homeomorphic to $\mathbb{C}$ and finally the proof is finished by Proposition 2.5.

3. Main result. First we want to describe the set of fixed points of a polynomial automorphism of $\mathbb{C}^{2}$. To do this, the following lemmas will be useful:

LEMMA 3.1. Let $X$ be an irreducible affine curve with infinite automorphism group. Then $X$ is either isomorphic to $\mathbb{C}^{*}$ or it is homeomorphic to $\mathbb{C}$. Moreover, in the last case $X$ can have at most one (necessarily irreducible) singularity.

Pr o of. Let $X_{0}$ be a normalization of $X$ and $X_{1}$ be a smooth completion of $X_{0}$. Since $\operatorname{Aut}(X)$ is infinite, so is $\operatorname{Aut}\left(X_{0}\right)$. The latter group is a subgroup of $\operatorname{Aut}\left(X_{1}\right)$ which stabilizes the divisor $D:=X_{1} \backslash X_{0}$. Since $D$ is ample, Aut $\left(X_{0}\right)$ must be linear (for details see [Jel3], 3.7). Moreover, since $\operatorname{Aut}\left(X_{0}\right)$ is infinite, $X_{1}$ is rational (see ibid., 3.12), i.e., $X_{1}=\mathbb{P}^{1}(\mathbb{C})$. Further, the singular points are permuted by polynomial automorphisms, hence the common number of points at infinity of $X_{0}$ and of points of $X_{1}$ which lie over the singular locus of $X$ must be at most two (in $X_{1}$ ). If there exist two such points we get either $X=X_{0}=\mathbb{C}^{*}$, or $X \neq X_{0}=\mathbb{C}$, and in the latter case $X$ has one irreducible singular point (which means that $X$ is homeomorphic to $\mathbb{C}$ ). If there is only one such point then $X=X_{0}=\mathbb{C}$.

Lemma 3.2. Let $\Gamma \subset \mathbb{C}^{2}$ be a curve with an irreducible equation $p(x, y)$ $=0$. Let $f \in \mathrm{Stab}_{\Gamma}$ be an element of infinite order. Then only two possibilities can occur:

1) There exists $s \in \mathbb{N}$ such that $f^{s}$ stabilizes all fibres of $p$. Moreover, $p$ is a $\mathbb{C}$ - or $\mathbb{C}^{*}$-family and $\Gamma$ is either homeomorphic to $\mathbb{C}$, or it is isomorphic to $\mathbb{C}^{*}$,

2) $\Gamma$ is the unique degenerate fibre of the family $p$ and it is homeomorphic to $\mathbb{C}$.

Proof. By the Hilbert Nullstellensatz we have $p \circ f=c p$, for some $c \in \mathbb{C}^{*}$. There are two cases possible:

1) $c$ has a finite order, i.e., $c^{s}=1$ for some $s \geq 1$,

2) $c$ has an infinite order.

1) We can assume $c=1$ and then we have $p-\lambda=(p-\lambda) \circ f$ for every $\lambda \in \mathbb{C}$. This means that all fibres of $p$ are stable under $f$. Since $f$ is of infinite 
order, a generic fibre has an infinite automorphism group. Since the generic fibre is smooth and irreducible it must be isomorphic either to $\mathbb{C}$ or to $\mathbb{C}^{*}$ (see Lemma 3.1).

In the first case, by Corollary 2.2, $p$ has no degenerate fibres, and in particular $\Gamma \cong \mathbb{C}$.

In the second case, by the same corollary, $p$ has exactly one degenerate fibre. If it is the fibre $p^{-1}(0)=\Gamma$ then by Proposition 2.1 we obtain $\Gamma=$ $\left\{(x, y) \in \mathbb{C}^{2}: x^{k}=y^{l},(k, l)=1\right\}$ in some coordinate system. If this fibre is not $\Gamma$ then $\Gamma$ is generic and isomorphic to $\mathbb{C}^{*}$.

2) In this case the fibre $\Gamma_{\lambda}:=p^{-1}(\lambda)$ is transformed under $f$ onto the fibre $p=c \lambda$. More generally, under $f^{r}$ this fibre goes onto the fibre $p=c^{r} \lambda$ for $r \in \mathbb{Z}$. Since $c$ is of infinite order, for $\lambda \neq 0$ the fibre $\Gamma_{\lambda}$ is isomorphic to an infinite set of other fibres. This means that $\Gamma_{\lambda}$ is a generic fibre for all $\lambda \neq 0$. Hence $p$ has at most one degenerate fibre, $\Gamma_{0}$. If $\Gamma_{0}$ is degenerate, by Proposition 2.7 we have $\Gamma_{0}=\left\{(x, y) \in \mathbb{C}^{2}: x^{k}=y^{l},(k, l)=1\right\}$ in some coordinate system.

If $p$ has no degenerate fibres at all, then Proposition 2.1 shows that $p$ is a family of $\mathbb{C}$-curves, and in particular $\Gamma \cong \mathbb{C}$.

Using the lemma above we describe the set Fix $f$ of fixed points of a polynomial automorphism $f$ of the affine plane. We have:

THEOREM 3.3. Let $f: \mathbb{C}^{2} \rightarrow \mathbb{C}^{2}$ be a non-trivial polynomial automorphism. Then the set $S=$ Fix $f$ is either finite, or a finite union of disjoint $\mathbb{C}$-curves.

Conversely, if $S$ is a finite subset of the plane, or a finite union of disjoint plane $\mathbb{C}$-curves, then there is $f \in \operatorname{Aut}\left(\mathbb{C}^{2}\right)$ such that $S=\operatorname{Fix} f$.

Proof. Let $\Gamma$ be a one-dimensional component of $S$. We show that in this case $\Gamma \cong \mathbb{C}$.

Since any automorphism of the affine plane of a finite order is conjugate to a linear one (see e.g. [Kam]), in which case there is nothing to prove, we can assume that $f$ is of infinite order. By Lemma 3.2 and Proposition 2.5 there are only two cases possible:

$1)$ in some coordinates $\Gamma=\left\{(x, y) \in \mathbb{C}^{2}: x^{k}=y^{l},(k, l)=1\right\}$,

2) $\Gamma$ is isomorphic to $\mathbb{C}^{*}$.

Now we will show that the first case is possible only if $k=1$ or $l=1$, and the second case is excluded. Indeed, the following lemma is true:

LEMMA 3.4. Let $f$ be a polynomial automorphism of the plane which stabilizes the curve $R=\left\{(x, y): x^{k}=y^{l},(k, l)=1\right\}$. Then each onedimensional component $\Gamma$ of Fix $f$ is isomorphic to $\mathbb{C}$.

Proof. Let $f=\left(f_{1}, f_{2}\right)$. Since $R$ is $f$-stable we have $f_{1}^{k}-f_{2}^{l}=c\left(x^{k}-y^{l}\right)$ 
for some non-zero constant $c$. Hence if $a:=\operatorname{deg} f_{1}$ and $b:=\operatorname{deg} f_{2}$ are both greater than 1 we have $\operatorname{deg} f_{1}^{k}=\operatorname{deg} f_{2}^{l}$. But it is well known (see [Kul]) that if $f$ is a polynomial automorphism then $\operatorname{deg} f_{1} \operatorname{divides} \operatorname{deg} f_{2}$ or vice versa, thus we can assume that $b=\lambda a$ for some non-zero integer $\lambda$. Hence $k a=l \lambda a$ and $k=l \lambda$. If $k \neq 1$ and $l \neq 1$ this is a contradiction.

Thus either " $a=1$ or $b=1$ ", or " $k=1$ or $l=1$ ".

Assume that $a=1$ or $b=1$. This means that one of the polynomials $f_{1}, f_{2}$, say $f_{1}$, is linear. We have $\Gamma \subset\left\{f_{1}-x=0\right\}$. Hence if $f_{1} \neq x$ then $\Gamma$ is a line. In the other case $f$ is a triangular automorphism, $f(x, y)=$ $(x, c y+p(x))$, and then Fix $f$ is known to be a finite union of $\mathbb{C}$-curves.

If $k=1$ or $l=1$ then $R$ is isomorphic to $\mathbb{C}$ and by the AbhyankarMoh-Suzuki theorem we can assume that $R=\{(x, y): x=0\}$. But in this case $f(x, y)=(c x, b y+p(x))$ and it is easy to see that a one-dimensional component of Fix $f$ is either a straight line or it has an equation $(b-1) y+$ $p(x)=0($ if $b \neq 1)$. In both cases $\Gamma$ is isomorphic to $\mathbb{C}$.

We proceed now with the proof of Theorem 3.3.

1) It follows immediately from the lemma that $\Gamma \cong \mathbb{C}$, i.e., $k=1$ or $l=1$.

2) We will show that the case $\Gamma \cong \mathbb{C}^{*}$ is impossible.

Let $p$ be an irreducible equation of $\Gamma$. Since $\Gamma$ is not homeomorphic to $\mathbb{C}$, Lemma 3.2 implies that $f^{s}$ stabilizes all fibres of $p$ for some $s \in \mathbb{N}$. We can assume that $s=1$. This means that all fibres of $p$ are stable under $f$ and the generic fibre is $\mathbb{C}$ or $\mathbb{C}^{*}$. By Corollary 2.2 the first case is impossible, hence the generic fibre must be $\mathbb{C}^{*}$. Hence $p$ has only one degenerate fibre. By Proposition 2.7 it cannot be the fibre over 0 . Let $w$ be the unique degenerate point and let $R$ be some irreducible component of $\Gamma_{w}$. Since $\Gamma_{w}$ has only a finite number of irreducible components the curve $R$ is stable under some iteration $F:=f^{r}$ of $f$. We have again two cases to consider:

(*) $R=\Gamma_{w}$,

(**) $R$ is a proper component of $\Gamma_{w}$.

(*) In this case $R$ is homeomorphic to $\mathbb{C}$ and stable under $F$ and by Lemma 3.4 we get $\Gamma \cong \mathbb{C}$, which is a contradiction.

(**) Let $R=\{q=0\}$ for some irreducible polynomial $q$. Since $R \neq$ $\{p=0\}$ we see that $\operatorname{deg} q<\operatorname{deg} p$, which shows that the families $p$ and $q$ are different. By Lemma 3.2 only two possibilites can occur: either all fibres of $q$ are stable under some iteration of $F$, or $R$ is homeomorphic to $\mathbb{C}$. The second possibility cannot occur, by Lemma 3.4 .

Hence we can assume that $F$ stabilizes all fibres of $q$. But $F$ is an iteration of $f$, hence it also stabilizes all fibres of $p$. Since a generic fibre of $q$ intersects a generic fibre of $p$ in at most $N=(\operatorname{deg} q)(\operatorname{deg} p)$ points, the order of $F$ is at most $N !=1 \cdot \ldots \cdot N$. This is a contradiction again. 
Hence case 2) is excluded and we have proved that if a curve $\Gamma$ is a component of Fix $f$ for a polynomial automorphism $f$ then $\Gamma$ is isomorphic to $\mathbb{C}$. Further in this case we can assume by the Abhyankar-Moh-Suzuki theorem (see Remark 2.6) that $\Gamma=\left\{(x, y) \in \mathbb{C}^{2}: x=0\right\}$. Since $f$ is the identity on $\Gamma$ we have $f=(c x, y+p(x))$ with $p(0)=0$. This means that Fix $f$ consists of one or more (disjoint) straight lines, in particular, it is of pure dimension. Hence Fix $f$ is either finite, or a union of disjoint $\mathbb{C}$-curves.

Now we prove the converse: if $S$ is a finite subset of the plane, or a finite union of disjoint plane $\mathbb{C}$-curves, then there is $f \in \operatorname{Aut}\left(\mathbb{C}^{2}\right)$ such that $S=\left\{x \in \mathbb{C}^{2}: f(x)=x\right\}$. Of course we can assume that $S$ is non-empty.

First assume that $S$ is finite. The following is proved in [Jel4]:

LemMA 3.5. Let $n \geq 2$ and $A=\left\{a_{1}, \ldots, a_{r}\right\}, B=\left\{b_{1}, \ldots, b_{r}\right\} \subset \mathbb{C}^{n}$, where $a_{i} \neq a_{j}$ and $b_{i} \neq b_{j}$ for $i \neq j$. Then there is a polynomial automorphism $F$ of $\mathbb{C}^{n}$ such that $F\left(a_{i}\right)=b_{i}, i=1, \ldots, r$.

Let $S=\left\{a_{1}, \ldots, a_{r}\right\}$ and suppose $F \in \operatorname{Aut}\left(\mathbb{C}^{2}\right)$ has the property that $F\left(a_{i}\right)=(i, 0), i=1, \ldots, r$. Let $B=\{(1,0),(2,0), \ldots,(r, 0)\}$. If we construct an automorphism $G$ with $B=\operatorname{Fix} G$ then $f=F^{-1} \circ G \circ F$ has $\operatorname{Fix} f=S$. It is easy to check that we can take for $G$ the automorphism

$$
G(x, y)=\left(x+y+\prod_{i=1}^{r}(x-i), y+\prod_{i=1}^{r}(x-i)\right)
$$

Now let $S$ be a finite union of disjoint plane curves, i.e., $S=\bigcup_{i=1}^{r} \Gamma_{i}$, where $\Gamma_{i} \cong \mathbb{C}$ and $\Gamma_{i} \cap \Gamma_{j}=\emptyset$. By the Abhyankar-Moh-Suzuki theorem (see Remark 2.6) we can reduce the problem (as above) to the case when $\Gamma_{1}=\{(x, y): x=0\}$. Then necessarily $\Gamma_{i}=\left\{(x, y): x=a_{i}\right\}$ for some non-zero distinct complex numbers $a_{i}, i=2, \ldots, r$. Indeed, the polynomial $x$ restricted to $\Gamma_{i}$ is a non-zero function on $\Gamma_{i}$, hence it is some constant $a_{i}$ and if $h_{i}$ is an irreducible equation of $\Gamma_{i}$ then $h_{i}$ divides $x-a_{i}$, i.e. $h_{i}=\operatorname{const}\left(x-a_{i}\right)$.

Thus $\Gamma_{i}=\left\{x=a_{i}\right\}, i=1, \ldots, r\left(\right.$ here $\left.a_{1}=0\right)$. Now it is easy to check that the automorphism

$$
G(x, y)=\left(x, y+\prod_{i=1}^{r}\left(x-a_{i}\right)\right)
$$

has $\operatorname{Fix} G=S$.

Now we use the above theorem to determine the one-dimensional identity sets in $\mathbb{C}^{2}$. Let us recall the definition:

Definition 3.6 (see [Jel1], [Jel2]). Let $\Gamma$ be an affine curve in $\mathbb{C}^{2}$. We say that $\Gamma$ is an identity set for polynomial automorphisms of $\mathbb{C}^{2}$ if any two polynomial automorphisms that coincide on $\Gamma$ must be equal. 
The following corollary generalizes some results from [M-W], [Jel1], [Jel2]:

Corollary 3.7. An affine curve $\Gamma \subset \mathbb{C}^{2}$ is an identity set for polynomial automorphisms of $\mathbb{C}^{2}$ if and only if it is not isomorphic to a union of disjoint $\mathbb{C}$-curves.

Proof. The condition is necessary by the last part of Theorem 3.3.

It is also sufficient. Indeed, suppose $\Gamma$ is not isomorphic to a union of disjoint $\mathbb{C}$-curves and let $f, g \in \operatorname{Aut}\left(\mathbb{C}^{2}\right)$ be two automorphisms that coincide on $\Gamma$. Then $\Gamma$ is a one-dimensional subset of Fix $F$ for the automorphism $F:=f \circ g^{-1}$, and by Theorem 3.3, $F$ must be trivial. Hence $f=g$ and we have proved that $\Gamma$ is an identity set.

Now we are in a position to describe irreducible affine curves with infinite group $\operatorname{Stab}_{\Gamma}$.

ThEOREM 3.8. Let $\Gamma \subset \mathbb{C}^{2}$ be an irreducible affine curve with $\mathrm{Stab}_{\Gamma}=$ $\left\{f \in \operatorname{Aut}\left(\mathbb{C}^{2}\right): f(\Gamma)=\Gamma\right\}$ infinite. Then only two cases are possible:

1) $\Gamma \cong \mathbb{C}^{*}$,

2) $\Gamma$ is homeomorphic to $\mathbb{C}$, i.e., in some coordinates $\Gamma=\left\{(x, y) \in \mathbb{C}^{2}\right.$ : $\left.x^{k}=y^{l},(k, l)=1\right\}$.

Proof. We can assume that $\Gamma$ is not isomorphic to $\mathbb{C}$. Hence $\Gamma$ is an identity set and consequently the restriction to $\Gamma$ gives the inclusion $\operatorname{Stab}_{\Gamma} \subset \operatorname{Aut}(\Gamma)$. Since $\operatorname{Stab}_{\Gamma}$ is infinite, so is $\operatorname{Aut}(\Gamma)$. By Lemma 3.1 and Proposition 2.5 the proof is finished.

We conclude this paper with the following theorem:

TheOREM 3.9. Let $\left\{\Gamma_{1}, \ldots, \Gamma_{s}\right\}$ be a finite family of non-rational curves in $\mathbb{C}^{2}$. Then the automorphism group of the variety $X:=\mathbb{C}^{2} \backslash \bigcup_{i=1}^{s} \Gamma_{i}$ is finite.

Proof. Let $f \in \operatorname{Aut}(X)$. By Corollary 54 in [Jel2] we can extend $f$ to the whole of $\mathbb{C}^{2}$ and consequently $\operatorname{Aut}(X)=\operatorname{Stab}_{\Gamma}$, where $\Gamma:=\bigcup_{i=1}^{s} \Gamma_{i}$. Moreover, if $H:=\operatorname{Stab}_{\Gamma} \cap \operatorname{Stab}_{\Gamma_{1}}$ then $(\operatorname{Aut}(X): H) \leq s$ and it is enough to show that $H$ is finite. But since $\Gamma_{1}$ is non-rational, $\operatorname{Stab}_{\Gamma_{1}}$ is finite according to Theorem 3.8 .

R emark 3.10. Wakabayashi (see [Iit2], pp. 15-16) gave conditions for the complement of a finite family of straight lines in $\mathbb{C}^{2}$ to have a finite automorphism group.

Acknowledgements. This paper was written during the author's stay at the University of Erlangen-Nürnberg. I would like to thank Professor Wolf Barth for the invitation and hospitality. I am also pleased to express my gratitude to T. Szemberg for helpful discussions. 


\section{References}

[A-M] S. Abhyankar and T. Moh, Embeddings of the line in the plane, J. Reine Angew. Math. 276 (1975), 148-166.

[Iit1] S. Iitaka, On logarithmic Kodaira dimension of algebraic varieties, in: Complex Analysis and Algebraic Geometry, Iwanami, Tokyo, 1977, 178-189.

[Iit2] —, Birational Geometry for Open Varieties, Les Presses de L'Université de Montréal, 1981.

[Jel1] Z. Jelonek, Identity sets for polynomial automorphisms, J. Pure Appl. Algebra 76 (1991), 333-339.

[Jel2] —, Irreducible identity sets for polynomial automorphisms, Math. Z. 212 (1993), 601-617.

[Jel3] —, Affine smooth varieties with finite group of automorphisms, ibid., to appear.

[Jel4] - , The extension of regular and rational embeddings, Math. Ann. 277 (1987), $113-120$.

[Jel5] - Sets determining polynomial automorphisms of $\mathbb{C}^{2}$, Bull. Polish Acad. Sci. Math. 37 (1989), 247-250.

[Kal] S. Kaliman, Polynomials on $\mathbb{C}^{2}$ with isomorphic generic fibers, Soviet Math. Dokl. 33 (1986), 600-603.

[Kam] T. Kambayashi, Automorphism group of a polynomial ring and algebraic group action on an affine space, J. Algebra 60 (1979), 439-451.

[Kul] W. van der Kulk, On polynomial rings in two variables, Nieuw Arch. Wisk. 1 (1953), 33-41.

[M-W] J. MacKay and S. Wang, An inversion formula for two polynomials in two variables, J. Pure Appl. Algebra 76 (1986), 245-257.

[Sak] F. Sakai, Kodaira dimension of complements of divisors, in: Complex Analysis and Algebraic Geometry, Iwanami, Tokyo, 1977, 239-257.

[Suz1] M. Suzuki, Propriétés topologiques des polynômes de deux variables complexes, et automorphismes algébriques de l'espace $\mathbb{C}^{2}$, J. Math. Soc. Japan 26 (1974), 241-257.

[Suz2] - Sur les opérations holomorphes du groupe additif complexe sur l'espace de deux variables complexes, Ann. Sci. Ecole Norm. Sup. 10 (1977), 517-546.

[Zai] M. G. Ză̌denberg, Isotrivial families of curves on affine surfaces and characterization of the affine plane, Math. USSR-Izv. 30 (1988), 503-532.

[Z-L] M. G. Zaĭdenberg and V. Ya. Lin, An irreducible simply connected algebraic curve in $\mathbb{C}^{2}$ is equivalent to a quasihomogeneous curve, Soviet Math. Dokl. 28 (1983), 200-203.

INSTITUTE OF MATHEMATICS

JAGIELLONIAN UNIVERSITY

REYMONTA 4

30-059 KRAKÓW, POLAND 